To appear in the Journal of Modern Optics

Vol. 00, No. 00, 10 January 2015, 1-7

10.1080/0950034YYXxxxxxxx

\title{
Structural colors of nickel bioreplicas of butterfly wings
}

\author{
Tomas Tolenis ${ }^{\mathrm{a}}$, Stephen E. Swiontek ${ }^{\mathrm{b}}$, and Akhlesh Lakhtakia ${ }^{\mathrm{b} *}$ \\ ${ }^{a}$ Center for Physical Sciences and Technology, Savanoriu ave. 231, LT-02300, Vilnius, Lithuania; \\ ${ }^{b}$ Pennsylvania State University, Department of Engineering Science and Mechanics, Nanoengineered \\ Metamaterials Group, University Park, PA 16802, USA \\ (xx September 2015)
}

\begin{abstract}
The two-angle conformally-evaporated-film-by-rotation technique (TA-CEFR) was devised to coat the wings of the monarch butterfly with nickel in order to form a 500-nm-thick bioreplica thereof. The bioreplica exhibits structural colors that are completely obscured in actual wings by pigmental colors. Thus, the TA-CEFR technique provides a way to replicate, study, and exploit hidden morphologies of biological surfaces.
\end{abstract}

Keywords: biomimetic optics; bioreplication; hidden structures; structural color

\section{Introduction}

Humans have had a long-standing fascination with colors for both beauty and diverse functionalities such as recognition and camouflage. Light either emanating from or scattered by an object has a physical spectrum that is captured by the eyes and interpreted by the brain. Colors of biological objects are very commonly due to chemical pigments and dyes [1]. Other colors have purely physical origins based on commonplace optical phenomena such as scattering, diffraction, and interference $[2,3]$. Often, complex color patterns have both physical and chemical origins [4].

Susceptible to environmental insults through exposure to high temperature, moisture, and sunlight, colors originating from chemicals alter and even fade over time [5]. In contrast, colors originating from physical mechanisms are environmentally stabler. Since light rays do not carry information on the mechanism of color production, our eyes cannot discriminate between colors based on their origin, tempting researchers to replace chemical colors by physical colors. An additional benefit may be the reduction in the release of hazardous volatile organic compounds during the manufacture and deployment of pigments and dyes.

Considerable progress has been made during the last two decades towards reproducing the iridescent blues of the wings of many butterfly species in the Morpho genus [6, 7]. Although granules of pigments such as biopterin are present in the scales of the wings of some Morpho species [8, 9], the iridescent blues are of physical origin. These colors result from a collaboration of three structural effects: multilayer interference, the diffraction-grating effect due to a periodic array of ridges on the scales on the wings, and the nonuniformity of the heights of those ridges [10].

In a biomimetic process to reproduce the structural features of the Morpho wing, first an irregularized surface grating is stamped on a resin film and then a periodic $\mathrm{SiO}_{2} / \mathrm{TiO}_{2}$ multilayer is deposited on the grating [6]. This process is suitable to colorize small surfaces, e.g., flakes that can be dispersed in paints, cosmetics, and security inks $[3,7]$. In a bioinspired process that excludes

${ }^{*}$ Corresponding author. Email: akhlesh@psu.edu 
both the surface grating and its irregularization, a periodic multilayer of two optically distinct polymers is cut up into yarn which is then embedded in a third polymer and flattened [11]. The composite yarn has been woven into non-pigmented but structurally colored fabrics that however lack the attractive iridescence of the Morpho blues [3].

Butterfly wings are so delicate that an actual wing is not used in either of the two foregoing processes. Yet the deposition of a nanoscale thin film on a butterfly wing ought to capture the surface structure of the wing. In a bioreplication [12] process, first a thin film of nickel was conformally deposited on the wing of a monarch butterfly (Danaus plexippus) and then the wing was plasmaashed away, leaving behind a bioreplica [13]. Whereas no pigmental contributions to the colors on the wing would be displayed by the nickel replica, some structural contributions to the wing colors should be. Multilayer interference would not contribute because the bioreplica is made of a single material, but surface gratings, whether strictly periodic or somewhat irregularly periodic, would.

The nickel thin film was deposited using the conformally-evaporated-film-by-rotation (CEFR) technique [13]. The wing was mounted on a planar substrate, the substrate was affixed to a platform that could rotate rapidly about a central normal axis passing through it, and the entire assembly was installed in a vacuum chamber. Also present in the vacuum chamber was a metal boat containing nickel that could be evaporated by passing an electric current through the boat. After suitably depressurizing the vacuum chamber, nickel was evaporated. A collimated portion of the nickel vapor was obliquely incident on the wing that was rapidly rotated during deposition. A $\sim 250-\mathrm{nm}-$ thick columnar thin film of nickel condensed conformally on the wing. However, structural colors were not visually evident on the bioreplica.

Hence, we devised the two-angle-CEFR (TA-CEFR) technique for better replication of the surface morphology. The nickel bioreplica of the D. plexippus hindwing turned out to display several colors dependent on the viewing angle. We report this development in this communication. Section 2 describes the sample preparation, the implementation of the TA-CEFR technique, and the imagery of the bioreplica. Section 3 presents and discusses the experimental results obtained. The communication ends in Sec. 4 with some concluding remarks.

Figure 1. Monarch (Danaus plexippus) butterfly.

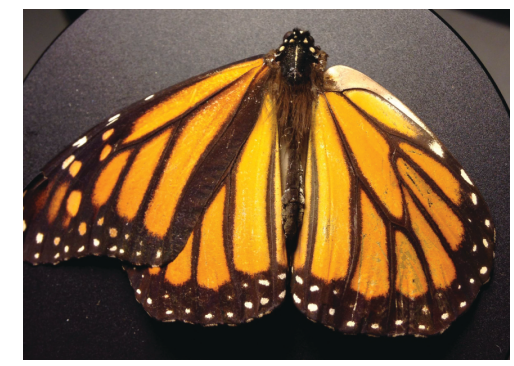

\section{Materials and Methods}

One notices from Fig. 1 that the wings of a monarch butterfly are dominated by orange sections separated by blackish lines and possess blackish fringes with whitish spots. The orange hues are caused by mixtures of pigments called pterins and ommochromes [14] present in the scales that the wings comprise [4]. The scales possess an array of long parallel ridges separated by crossribs, much like a ladder. These crossribs ought to function as surface gratings, but any consequent structural colors are completely overshadowed by the pigmental colors.

The dead D. plexippus specimen used by us was donated by Folk's Butterfly Farm (Nescopeck, PA, USA). The left hindwing (Fig. 2(a)) of the specimen was removed and was used as the sample. It was first washed with ethanol to remove particulates and then dried under the intense light of a tungsten light bulb. Next, the sample was mounted on a borosilicate-glass substrate via copper 

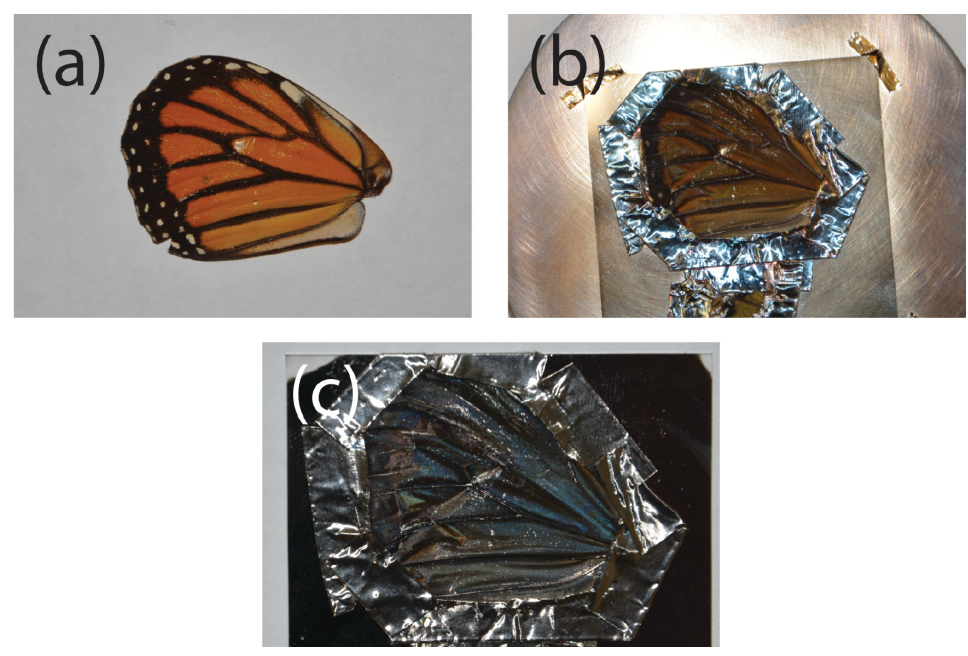

Figure 2. Photographs of the left hindwing (a) before the implementation of the TA-CEFR technique, (b) after the first nickel thin film was deposited with the collimated vapor directed at angle $\chi_{v}=7^{\circ}$, and (c) after the second nickel thin film was deposited with the collimated vapor directed at angle $\chi_{v}=20^{\circ}$.

tape (Uline, Pleasant Prairie, WI, USA). Finally, the entire assembly was mounted on a planar platform using Kapton ${ }^{\circledR}$ tape (Uline, Pleasant Prairie, WI, USA). This tape can withstand high temperature, produces negligible outgassing during the deposition, and maintains its adhesive capabilities throughout that process.

The planar platform with the sample and the glass substrate was mounted on the axle of a computer-controlled stepper motor inside a custom-built vacuum chamber equipped to perform resistive-heating physical vapor deposition. The chamber contained a $3.2-\mathrm{mm}$ wide tungsten trough (S4-.005W, R. D. Mathis, Long Beach, CA, USA) in which intertwined strands of nickel wire (99.6\% pure, $0.5 \mathrm{~mm}$ diameter) were placed. The distance between the sample and the nickel strands was fixed at roughly $15 \mathrm{~cm}$. The chamber was closed and the pressure was reduced to $\sim 50 \mu$ Torr. The stepper motor was turned on to rotate the sample at $120 \mathrm{rpm}$ about the axle. A second computercontrolled stepper motor then oriented the platform so that during deposition the collimated vapor of nickel would arrive at an angle $\chi_{v}=7^{\circ}$ with respect to the platform plane.

About 87-A current was then passed through the tungsten trough to generate the nickel vapor. A quartz crystal monitor mounted close to the sample was used to maintain the deposition rate at $\sim 1 \mathrm{~nm} \mathrm{~s}^{-1}$. The deposition lasted until a $\sim 250$-nm-thick film of nickel was deposited. Then the current was switched off, the motors were stopped, and the chamber was exposed to the atmosphere.

The foregoing process was repeated to deposit another columnar thin film of nickel on top of the first one. Whereas the thickness of the second thin film was also $\sim 250 \mathrm{~nm}$, it was deposited with the angle $\chi_{v}=20^{\circ}$. Removal of the wing by plasma ashing was not undertaken as it is unnecessary for the optical investigation of the nickel bioreplica.

The bioreplica structure was analyzed with a scanning electron microscope (SEM) (FEI Nova NanoSEM 630, Hillsboro, Oregon, USA). A small piece of the coated hindwing was cut off for analysis. Since the nickel film is conductive, no additional coating by gold (or some other metal) was necessary to prevent charging of the bioreplica by the impinging electrons during imaging.

Structural colors were imaged by a Nikon D90 camera with a $105-\mathrm{mm}$ lens. The schematic of the experimental setup is shown in Fig. 3. A 7-W 330-lumen compact fluorescent light (CFL) bulb was chosen as the light source since it is a common white-light source for indoor use. The bioreplica was mounted on a rotation stage, and the angle of incidence of white light was fixed at $70^{\circ}$ with respect to the normal to the mean plane of the bioreplica. The angle $\psi \in\left[-40^{\circ}, 20^{\circ}\right]$ between the camera and the normal to the mean plane of the bioreplica was changed in steps of $10^{\circ}$. At each value of $\psi \in\left\{-40^{\circ},-30^{\circ}, \ldots, 10^{\circ}, 20^{\circ}\right\}$, the bioreplica was photographed with the CFL bulb turned 
Figure 3. Schematic of the system used for photographing structural colors. A CFL bulb was used to illuminate the bioreplica from a direction at $70^{\circ}$ with respect to the normal to the mean plane of the bioreplica. A camera was mounted on a stepper motor. The angle $\psi \in\left[-40^{\circ}, 20^{\circ}\right]$ between the camera and the normal to the mean plane of the bioreplica was changed in steps of $10^{\circ}$.

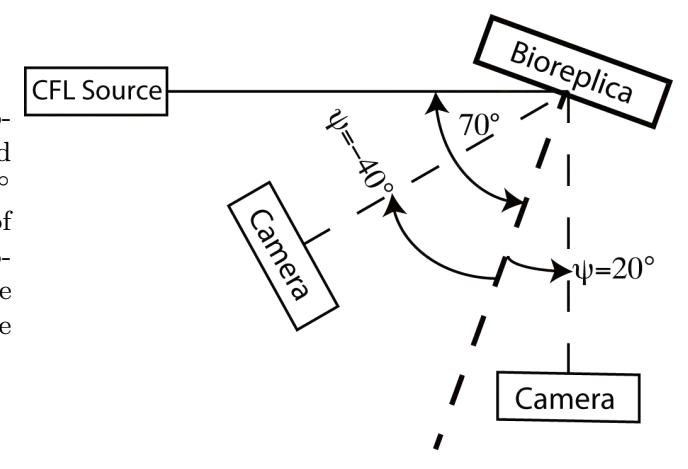

Figure 4. (a) SEM image of the nickel bioreplica showing the top surfaces of the scales of the wings replicated in nickel. (b) SEM image of the crossribs in the bioreplica.

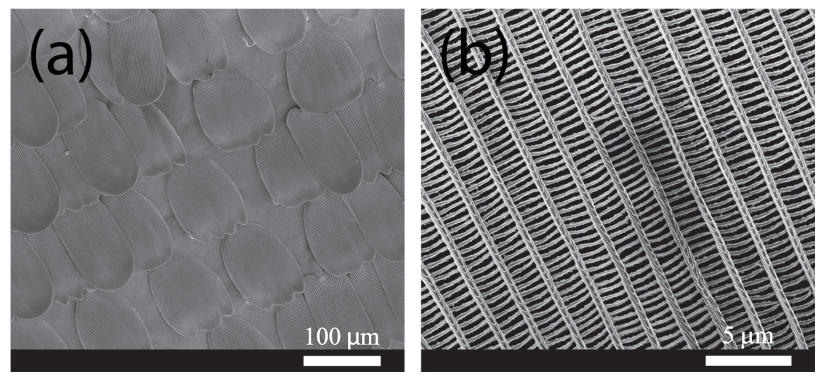

on.

\section{Results and Discussion}

The first deposition (with $\chi_{v}=7^{\circ}$ ) would lead to the formation of highly slanted columns of nickel, whereas the second deposition (with $\chi_{v}=20^{\circ}$ ) would lead to the formation of less slanted columns. This TA-CEFR method was devised to allow nickel vapor during the first deposition to penetrate deep into the butterfly wing and thus replicate the surface structure with higher fidelity during the second deposition.

After the first deposition, the native orange hues of the hindwing, although muted, were still evident through the nickel thin film, as captured by the photograph in Fig. 2(b). After the second deposition, the orange hues were replaced by a whole gamut of colors that were obvious to the naked eye. These colors can be seen in the photograph in Fig. 2(c). The colors were different from different viewing directions, as is characteristic of colors arising from diffraction by surface gratings $[2,3]$.

Figure 4 provides two SEM images of the nickel bioreplica. The wing is made up of scales of lateral dimensions $\sim 100 \mu \mathrm{m}$. Each scale has parallel ridges spaced about $2.2 \mu \mathrm{m}$ apart. The crossribs between the ridges are spaced about $500 \mathrm{~nm}$ apart. Diffraction gratings with periodicity of $500 \mathrm{~nm}$ can produce all visible colors via low-order diffraction. Using standard data for nickel [15], we determined that the specular reflectance of a 500-nm-thick nickel film for normally incident light varies from about $50 \%$ (at free-space wavelength $\lambda_{o}=400 \mathrm{~nm}$ ) to about $70 \%$ (at $\lambda_{o}=800 \mathrm{~nm}$ ). Therefore, the nickel bioreplica can function as a reflection grating.

To observe all the colors exhibited by the bioreplica, a small piece of it was removed and mounted on a rotational stage. By fixing the angle of the light source at $70^{\circ}$ from the normal to the mean plane of the piece of the bioreplica and changing the angle of observation, colors were captured by the Nikon D90 camera (Fig. 3). As $\psi$ decreased from $20^{\circ}$ to $-40^{\circ}$, the captured colors changed from blackish-violet to blue to bluish green to greenish yellow to copper and finally to orangish red overlaid by violet and blue, as may be gathered from Fig. 5 .

In order to confirm that the captured colors were instigated by the crossribs of the wing scales 
Figure 5. Colors of the nickel bioreplica captured by the camera at different values of the angle $\psi$ : (a) $20^{\circ}$, (b) $10^{\circ}$, (c) $0^{\circ}$, (d) $-10^{\circ}$, (e) $-20^{\circ}$, (f) $-30^{\circ}$, and $(\mathrm{g})-40^{\circ}$.
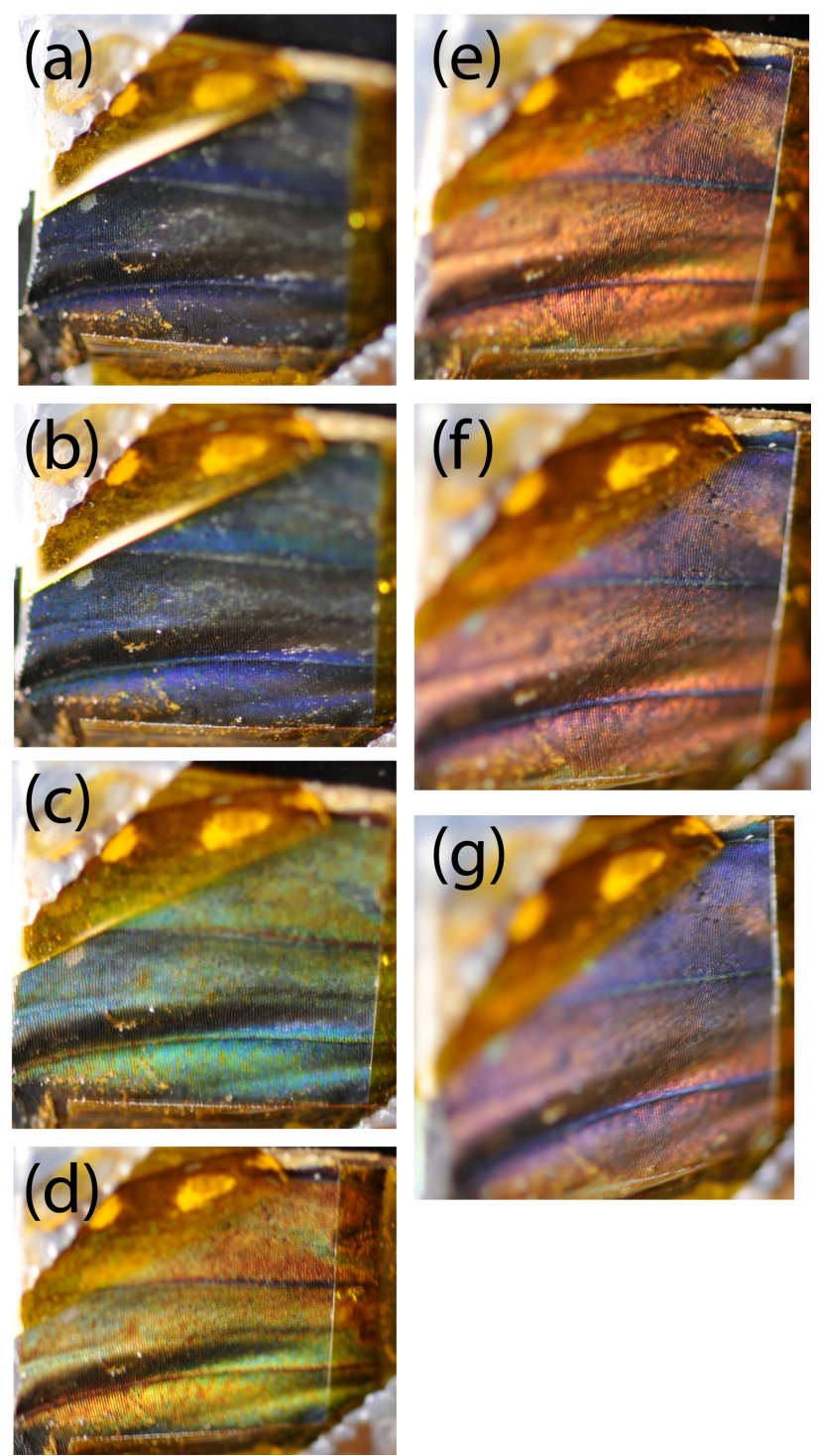

Table 1. $\lambda_{o}$ as a function of $\psi$ when $d=500 \mathrm{~nm}$ and $\psi_{r e f}=70^{\circ}$ in Eq. (1). Boldface values lie in the visible regime.

\begin{tabular}{|c|c|c|}
\hline$\psi$ & $m=-1$ & $m=-2$ \\
\hline \hline $20^{\circ}$ & $299 \mathrm{~nm}$ & $149 \mathrm{~nm}$ \\
\hline $10^{\circ}$ & $\mathbf{3 8 3} \mathrm{nm}$ & $192 \mathrm{~nm}$ \\
\hline $0^{\circ}$ & $\mathbf{4 7 0} \mathrm{nm}$ & $235 \mathrm{~nm}$ \\
\hline$-10^{\circ}$ & $\mathbf{5 5 7} \mathrm{nm}$ & $278 \mathrm{~nm}$ \\
\hline$-20^{\circ}$ & $\mathbf{6 4 1} \mathrm{nm}$ & $320 \mathrm{~nm}$ \\
\hline$-30^{\circ}$ & $\mathbf{7 2 0} \mathrm{nm}$ & $360 \mathrm{~nm}$ \\
\hline$-40^{\circ}$ & $791 \mathrm{~nm}$ & $\mathbf{3 9 6} \mathrm{nm}$ \\
\hline
\end{tabular}

replicated by the nickel film, the diffraction wavelength

$$
\lambda_{o}(\psi)=\frac{d}{m}\left(\sin \psi-\sin \psi_{r e f}\right), \quad m \in\{0, \pm 1, \pm 2, \ldots\}
$$


was calculated as a function of $\psi$, with $m$ as the diffraction order, $d$ as the average spacing between two successive crossribs, and $\psi_{\text {ref }}$ as the value of $\psi$ corresponding to specular reflection. We set $d=500 \mathrm{~nm}$ according to measurements made on the SEM images and $\psi_{\text {ref }}=70^{\circ}$ according to the experimental setup shown in Fig. 3.

The diffraction wavelengths $\lambda_{o}(\psi)$ are listed in Table 1 for $m \in\{-1,-2\}$. The boldface entries in this table lie within the spectral regime visible to most humans. Due to irregularities in the inter-crossrib spacing as well as the nonplanarity of the wing, every value of $\lambda_{o}(\psi)$ provided in Table 1 is not to be considered as precise but as representative of a small range. When $\psi=20^{\circ}$, the nickel bioreplica looks blackish violet in Fig. 5(a). The bluish color in Fig. 5(b) for $\psi=10^{\circ}$ is matched by $\lambda_{o}=383 \mathrm{~nm}$ for $m=-1$, the bluish green color in Fig. $5(\mathrm{c})$ for $\psi=0^{\circ}$ by $\lambda_{o}=470 \mathrm{~nm}$ for $m=-1$, the greenish yellow color in Fig. 5(d) for $\psi=-10^{\circ}$ by $\lambda_{o}=557 \mathrm{~nm}$ for $m=-1$, the copper color in Fig. 5(e) for $\psi=-20^{\circ}$ by $\lambda_{o}=641 \mathrm{~nm}$ for $m=-1$. The orangish red color in Fig. 5(f) for $\psi=-30^{\circ}$ is matched by $\lambda_{o}=720 \mathrm{~nm}$ for $m=-1$, and the violet overlay in the same figure by $\lambda_{o}=360 \mathrm{~nm}$ for $m=-2$. Finally, the orangish red color in Fig. 5(f) for $\psi=-40^{\circ}$ is overlaid by a blue color that is matched by $\lambda_{o}=396 \mathrm{~nm}$ for $m=-2$. Thus, the viewing-angledependent colors displayed by the bioreplica are structural colors produced by the diffraction of light by the (almost) periodically spaced crossribs.

\section{Concluding Remarks}

The two-angle-CEFR technique was devised to coat nickel on the hindwing of a monarch butterfly and thereby replicate its surface morphology. Structural colors observed on the 500-nm-thick nickel bioreplica were engendered by the periodic arrays of crossribs on the wing scales. These structural colors, which are completely hidden by pigmental colors in the monarch-butterfly wings, thereby were uncovered. Thus, the TA-CEFR technique provides a way to replicate, study, and exploit hidden morphologies of biological surfaces.

In closing, let us note that the physical basis of structural color can also be exposed to varying degrees of success by other bioreplication techniques [12] such as the solution-based infiltration methods [16, 17], electroless deposition [18], and atomic layer deposition [19]. Electroless deposition and infiltration methods are suitable for volumetric structures, whereas atomic layer deposition is suitable for areal structures, but none of these three techniques is likely to be industrially scalable. In contrast, by thickening the TA-CEFR nickel film via electroless deposition [20], one could make robust molds to stamp sheets and fabrics to exhibit structural colors. We note that a multi-angleCEFR technique may have to be devised for better bioreplication of certain specimen. Furthermore, the stamped sheet or fabric may be made of a carefully selected material so that functionalities in addition to structural coloration could be realized [21].

Acknowledgments. This work was partially supported by the project Promotion of Student Activities (VP1-3.1-ŠMM-01-V-02-003) from the Research Council of Lithuania to TT. This project is funded by the Republic of Lithuania and European Social Fund under the 2007-2013 Human Resources Development Operational Programme's priority 3. Additional funding was provided to AL and SES by the Charles Godfrey Binder Endowment at the Pennsylvania State University.

\section{References}

[1] Lee, D. Nature's Palette: The Science of Plant Colors; University of Chicago Press: Chicago, 2007.

[2] Vigneron, J.-P.; Simonis, P. In Advances in Insect Physiology. Vol. 38: Insect Integument and Colour; Casas, J.; Simpson, S.J., Eds.; Elsevier: Oxford, 2010; Chapter 5. 
[3] Dushkina, N.; Lakhtakia, A. In Engineered Biomimicry; Lakhtakia, A.; Martín-Palma, R.J., Eds.; Elsevier: Waltham, 2013; Chapter 11.

[4] Ghiradella, H. In Advances in Insect Physiology. Vol. 38: Insect Integument and Colour; Casas, J.; Simpson, S.J., Eds.; Elsevier: Oxford, 2010; Chapter 4.

[5] Wicks Jr., Z.W.; Jones, F.N.; Pappas, S.P.; Wicks, D.A. Organic Coatings: Science and Technology, 3rd ed.; Wiley: Hoboken, 2007.

[6] Saito, A.; Miyamura, Y.; Ishikawa, Y.; Murase, J.; Akai-Kasaya, M.; Kuwahara, Y. Proc. SPIE 2009, 7205, 720506.

[7] Saito, A.; Murase, J.; Yonezawa, M.; Watanabe, H.; Shibuya, T.; Sasaki, M.; Ninomiya, T.; Noguchi, S.; Akai-Kasaya, M.; Kuwahara, Y. Proc. SPIE 2012, 8339, 83390C.

[8] Kumazawa, K.; Tabata, H. Zool. Sci. 1996, 13, 843-847.

[9] Tabata, H.; Hasegawa, T.; Nakagoshi, M.; Takikawa, S.; Tsusue, M. Experientia 1996, 52, 85-87.

[10] Kinoshita, S.; Yoshioka, S.; Kawagoe, K. Proc. R. Soc. Lond. B 2002, 269, 1417-1421.

[11] Iohara, K.; Yoshimura, M.; Tabata, H.; Shimizu, S. Chem. Fibers Int. 2000, 50, 38-39.

[12] Pulsifer, D.P.; Lakhtakia, A. Bioinsp. Biomim. 2011, 6, 031001.

[13] Pulsifer, D.P.; Lakhtakia, A.; Martín-Palma, R.J.; Pantano, C.G. Proc. SPIE 2011, $7975,797500$.

[14] Davis, A.K.; Chi, J.; Bradley, C.; Altizer, S. PLoS One 2012, 7, e41323.

[15] Ordal, M.A.; Long, L.L.; Bell, R.J.; Bell, S.E.; Bell, R.R.; Alexander Jr., R.W.; Ward, C.A. Appl. Opt. 1983, 22, 1099-1119.

[16] Li, B.; Zhou, J.; Zong, R.; Fu, M.; Bai, Y.; Li, L.; Li, Q. J. Am. Ceram. Soc., 2006, 89, $2298-2300$.

[17] Tan, Y.; Zang, X.; Gu, J.; Liu, D.; Zhu, S.; Su, H.; Feng, C.; Liu, Q., Lau, W.M.; Moon, W.-J.; Zhang, D. Langmuir 2011, 27, 11742-11746.

[18] Tan, Y.; Gu, J.; Zang, X.; Xu, W.; Shi, K.; Xu, L.; Zhang, D. Angew. Chem. Int. Ed. Engl. 2011, 50, $8307-8311$.

[19] Huang, J.; Wang, X.; Wang, Z.L. Nano Lett., 2006, 6, 2325-2332.

[20] Sudagar, J.; Lian, J.; Sha, W. J. Alloys Compounds 2013, 571, 183-204.

[21] Lakhtakia, A. Bioinsp. Biomim. Nanobiomater. 2015, 4, 168-173. 